\title{
Teknokultura
}

ISSNe: 1549-2230

http://dx.doi.org/10.5209/TEKN.59548

\section{Alianzas de actores y construcción de una arena pública a través de la estrategia transmedia. Análisis del proyecto Chicas Nuevas 24 Horas $^{1}$}

\author{
Vanesa Saiz Echezarreta² ${ }^{2}$ Lidia Peralta García ${ }^{3}$; Elena Martínez Pérez ${ }^{4}$
}

Recibido: 6 de abril de 2018 / Revisado: 14 de junio de 2018 / Revisado: 21 de agosto de 2018 / Aceptado: 13 de septiembre de 2018 Open peer reviews

Resumen. En este artículo reflexionamos sobre el impacto de las estrategias transmedia como recurso para escenificar (visibilizar), captar la atención y acceder al espacio público mediatizado en la controversia sobre el estatuto de la prostitución y las formas de intervención en la lucha contra la trata con fines de explotación sexual en España. El principal objetivo reside en proponer un mapeo de actores (agentes institucionales, sociedad civil y actores mediáticos) en torno al proyecto Chicas Nuevas 24 horas $(\mathrm{CHN} 24 \mathrm{H})$, de la cineasta y activista Mabel Lozano. Para ello, observamos, mediante un ejercicio de etnografía multisituada bajo el enfoque de seguimiento del conflicto (follow the conflict) definido por George E. Marcus (1995), la red de alianzas participativas y los espacios de mediación en la configuración de una arena pública (Cefaï, 2011). El recorrido analítico nos conduce desde las primeras colaboraciones de Lozano con organizaciones especializadas, como la Asociación para la Prevención, Reinserción y Atención a la Mujer Prostituida (APRAMP) y la Red Española contra la trata, en 2013, hasta la celebración del Día Internacional contra la trata con fines de explotación sexual en 2017, en la que el Ministerio de Asuntos Sociales asume el proyecto CHN24H como propio. El grafo del entramado transmedia analizado ha priorizado la expansión del discurso frente a su profundización, y la lógica de la "sensibilización" en detrimento de la participación y la orientación a la acción.

Palabras clave: controversia; mediatización; prostitución; transmedia; trata con fines de explotación sexual.

\section{[en] Alliances of actors and construction of a public arena through transmedia strategy. Analysis of the New Girls 24 Hours project}

\footnotetext{
Abstract. In this article we reflect on the impact of transmedia strategies as a resource to stage (make visible), capture attention and access the public space. We do it in the frame of the controversy about the status of prostitution and the forms of intervention in the fight against sexual exploitation trafficking in Spain. The main objective lies in proposing a mapping of actors (institutional agents, civil society and media actors) of the project Chicas Nuevas 24 Horas (New Girls 24 hours, CHN24H), by the filmmaker and activist Mabel Lozano. We observe, through a multisite ethnography exercise, under the follow the conflict approach defined by George E. Marcus (1995), the network of participative alliances and mediation spaces in the configuration of a public arena (Cefaï, 2011). The analytical journey leads us from the first collaborations of Lozano with specialized organizations, such as the Association for the

1 Esta publicación se enmarca en el Proyecto $\mathrm{I}+\mathrm{D}+\mathrm{i}$ "Problemas públicos y controversias: diversidad y participación en la esfera mediática" (CSO2017-82109-R), financiado por el Ministerio de Economía y Competitividad.

2 Universidad de Castilla-La Mancha (España)

E-mail: vanesa.saiz@uclm.es

3 Universidad Castilla-La Mancha (España)

E-mail: lidia.peralta@uclm.es

$4 \quad$ Universidad de Castilla-La Mancha (España)

E-mail: elena.martinez.perez@hotmail.com
} 
Prevention, Reintegration and Attention to Prostituted Women (APRAMP) and the Spanish Network against Trafficking, in 2013, until the celebration of the International Day against trafficking for sexual exploitation in 2017, in which the Ministry of Social Affairs assumes the CHN24H project as its own. The graph of the transmedia network analyzed has prioritized the expansion of discourse instead of its deepening, and the logic of "sensitization" to the detriment of participation and orientation to action.

Keywords: controversy; mediatization; prostitution; trafficking; transmedia.

Sumario. 1. Introducción. 2. Objetivo, preguntas de investigación e hipótesis. 3. Metodología. 4. Resultados. 4.1. Mapeo de productos y actores. 4.2. Análisis de los elementos paratextuales de la estrategia transmedia. 5 Discusión: mediatización de la controversia y lógica transmedia. 6. Conclusiones. 7. Referencias.

Cómo citar: Saiz Echezarreta, V.; Peralta García, L. y Martínez Pérez, E. (2018). Alianzas de actores y construcción de una arena pública a través de la estrategia transmedia. Análisis del proyecto Chicas Nuevas 24 Horas, en Teknokultura 15(2), 207-222.

\section{Introducción}

La transformación de los problemas sociales en problemas públicos, entendidos como asuntos de interés común y compartido que son objeto de deliberación democrática y sobre los que se exige intervención de los poderes públicos (Gusfield, 1991) se adapta a las dinámicas de mediatización que configuran la actual esfera pública (Hjarvard, 2016; Peñamarín, 2017). En esta transformación interviene la transmedialidad, una lógica mediática que favorece la emergencia de alianzas entre actores y la conexión de arenas públicas.

La prostitución ha sido conceptualizada históricamente como un problema público controvertido que reclama la intervención de las instituciones, y en el que se debate desde posiciones divergentes (Juliano, 2005; Heim, 2012; Gimeno, 2012). Actualmente, esta disputa se ha desplazado y abarca además de la discusión sobre la conceptualización y el diseño de políticas públicas destinadas a la prostitución, los debates en torno al fenómeno de la trata con fines de explotación sexual (definición, causas, cómo opera, así como los mecanismos más adecuados para combatirla) (Andrijasevic y Mai, 2016; Serughetti, 2018; Wagenaar, 2017). Nos interesa esta controversia por la condición liminal de la prostitución, es decir, porque los debates al respecto expresan las fisuras y las dimensiones problemáticas interseccionales de los imaginarios sociosexuales (Justo von Lurzer, 2014, pp. 7-14), la organización social de la sexualidad en un marco postcolonial (Mai, 2014) y cuestionan las concepciones neoliberales sobre la identidad, la autonomía y la libertad sexual (Sabsay, 2016, pp. 118-120).

Cuando se aborda la trata en el espacio público los ejercicios de representación actúan como espacios de focalización y orientación de los posicionamientos en conflicto, que van más allá del consenso sobre el reconocimiento de la importancia de la trata, su carácter delictivo y la vulneración de los derechos humanos que representa. Tal como señalan estudios previos (Devillard y Le Saulnier, 2015; Patte, 2006), los marcos y posicionamientos hegemónicos sobre la trata actúan como condición de posibilidad de la participación en la controversia general ligada a la prostitución. $\mathrm{Al}$ tener un "efecto de campo", permite a los actores acumular y hacer circular los capitales simbólicos, culturales y económicos. Tanto a nivel internacional como nacional, la trata está experimentando un proceso de emergencia en cuanto problema público sobre el que se exige intervención a las instituciones políticas y sociales, así 
como al conjunto de la ciudadanía. En España se encuentra vigente el II Plan Integral contra la trata de mujeres y niñas con fines de explotación sexual (2015-2018), que recoge la necesidad de aumentar la sensibilización social sobre este asunto y fomentar el rechazo a esta vulneración de los derechos fundamentales. El plan prevé que las acciones mediáticas sirvan en las tareas de prevención, detección e identificación de potenciales víctimas de trata. También se plantea que los medios de comunicación pueden ser claves en la desincentivación de la demanda de servicios sexuales, una de las prioridades de las políticas públicas a nivel internacional.

Los actores que participan de la controversia, visibilizan y escenifican su punto de vista y sus relatos sobre el asunto, con los que tratan de captar la atención y movilizar a públicos que potencialmente pueden adquirir compromisos con el problema planteado desde la perspectiva propuesta (Cefaï, 2016, pp. 27-28). Cada vez es mayor el consenso para identificar la trata como una manifestación de las violencias machistas y para establecerla como prioridad de las políticas feministas, como puede comprobarse en el texto refundido del Pacto de Estado contra la Violencia de Género (Gobierno de España, 2018). Sin embargo, la existencia de consenso en torno a la trata como expresión de la violencia patriarcal no elimina su carácter controvertido cuando se abordan cuestiones específicas y especialmente cuando se dirime su conexión con la prostitución. Estos debates suceden en un espacio continuo lleno de matices en el que participan múltiples voces y perspectivas: desde posturas abolicionistas que defienden que la práctica de la prostitución es siempre un modo de explotación sexual, y por tanto ha de ser erradicada; hasta las que defienden la regulación del trabajo sexual y reivindican la diferenciación entre prostitución forzada y no forzada. No obstante, este espacio complejo y diverso ha quedado en gran medida reducido en su traducción mediática.

Las posibilidades de diversificación y pluralidad en la esfera pública mediatizada (en adelante EPM) no restan peso a las dinámicas del poder jerarquizado, puesto que en las controversias los actores participan con capitales y recursos desiguales. Actualmente, la posición hegemónica en el asunto de la prostitución se identifica en un porcentaje significativo con el movimiento abolicionista y sus promotores institucionales, que parecen tener más capacidad para ganar espacio como voz privilegiada y legítima a la hora de definir los términos de la controversia y sus soluciones potenciales. Uno de los factores clave para ello es la alianza entre actores del activismo social, de la industria mediática y de las instituciones del Estado (Devillard y Le Saulnier, 2015). Esta posición adquiere poder para definir el horizonte de expectativas sobre el que resolver la controversia acerca del estatuto de la prostitución y el trabajo sexual en nuestras sociedades y además se le concede mayor capacidad para incidir en el diseño de las políticas públicas.

En cuanto a la visibilización y representación mediática de la trata, esta responde en las últimas décadas a unas pautas representacionales bastante homogéneas, como señalan investigaciones nacionales e internacionales (Andrijasevic y Mai, 2016; Kapur, 2002; Puñal Rama y Tamarit, 2017; Justo von Lurzer, 2014; Saiz-Echezarreta et al., 2018). En estas formas de representación hegemónicas destaca el recurso al realismo, la enunciación casuística y la centralidad del testimonio, junto con una retórica de la denuncia construida sobre lugares comunes consensuados y relatos estereotipados a partir de la mirada postcolonial. Esta combinación se acompaña en muchos casos de una presentación despolitizada del asunto y una fetichización de las víctimas, lo que deriva en los procesos de revictimización ya descritos en las investigaciones sobre las representaciones de las violencias machistas en la cultura popular (Núñez Puente y Fernández Romero, 2015). 


\section{Objetivo, preguntas de investigación e hipótesis}

En el marco de un proyecto sobre la incidencia de los procesos de mediatización en el debate público, desarrollamos una indagación etnográfica multisituada (Marcus, 1995) sobre la controversia en torno a la prostitución y la trata con fines de explotación sexual en la que se prioriza el examen de la circulación y articulación de significados culturales, objetos e identidades a través de diferentes arenas públicas y productos mediáticos. Este mapeo etnográfico busca nuevos locus de enunciación sobre la violencia y el cambio social que actúen dentro del paradigma del testimonio ético, es decir, que constituyan prácticas comunicativas que superando la lógica del reconocimiento se orienten hacia la responsabilidad política que tenemos como testigos y a la reparación del daño, así como que conecten las representaciones de la experiencia singular con las condiciones estructurales (Gámez Fuentes y Gómez Nicolau, 2017).

El estudio de caso analiza el proyecto transmedia liderado por Mabel Lozano Chicas Nuevas 24 horas $(\mathrm{CHN} 24 \mathrm{H})$. A partir de este proyecto y siguiendo sus desbordes, nos aproximamos al mapeo de actores (activistas, institucionales y mediáticos) y al entramado de interacciones entre estos, que participan en la conformación y legitimación de una posición hegemónica de orientación abolicionista en el espacio público, gracias a los recursos compartidos (institucionales, económicos, tecnológicos o de socialización) y a la visibilización e institucionalización de una perspectiva común (inscrita en un mundo transmediático que, como veremos, supera el proyecto original).

Frente al concepto de narrativa transmedia (Jenkins, 2011), utilizamos el de mundo transmediático (Klastrup y Tosca, 2004) puesto que se adapta mejor a las características de los discursos no ficcionales (información de actualidad, activismo, etc.) (Rodríguez Ferrándiz y Peñamarín, 2014). En esta conceptualización, la articulación de productos va más allá del modelo multiplataforma y no se define por su carácter narrativo, sino que, perteneciendo a cualquier género, los productos se articulan entre sí para crear sistemas de contenido abstracto o "imágenes mentales compartidas" que pueden tener coherencia y continuidad gracias a los sistemas de sentido (y el relato, si lo hubiere) que dicho mundo presupone. Generalmente, un mundo transmediático presenta una obra núcleo que condensa la propuesta narrativa y retórica que funciona como origen y da sentido al horizonte de expectativas políticas, axiológicas y afectivas, así como a las propuestas de acción colectiva derivadas de este; es decir, funciona como base de su justificación.

El objetivo es identificar cómo la lógica transmedia construye un mundo transmediático y a través de él se interviene en el desarrollo de una controversia abierta. Observamos cómo las alianzas entre actores y la focalización de un marco específico, centrado en la lucha contra la trata desde una perspectiva abolicionista (y victimocéntrica) inscrita en dicho mundo, podrían determinar las condiciones de configuración e inteligibilidad de la arena pública sobre prostitución y trata.

Nuestras preguntas de investigación contienen las siguientes inquietudes: ¿Qué estructura ha ido tomando a lo largo del tiempo el grafo transmedia del proyecto CHN24H? ¿Quiénes son los actores que forman parte del grafo y qué relación existe entre ellos? ¿Cómo sus alianzas participan de la conformación de la arena pública sobre prostitución y trata? ¿Qué rol juega la transmedialidad? ¿Contribuye a ampliar puntos de vista o reincide en un mismo mensaje ampliado por varios canales? Así mismo, apuntaremos brevemente una línea de indagación sobre la función del testimonio en la escenificación del mundo transmediático compartido por estos actores. 
Nuestra hipótesis es que en los procesos de mediatización de la trata esta construcción de hegemonía responde a varios factores: la emergencia y estabilización de redes organizacionales y personales en torno a dichos procesos; la utilización de una estrategia transmedia como herramienta para ofrecer coherencia, imbricar los discursos y elaborar un mundo transmediático que dé explicación al fenómeno, posicione a los actores implicados y justifique las propuestas de solución y de acción social y política; y, por último, el recurso de la narrativización del testimonio como hilo conductor del marco transmediático propuesto -comprendido principalmente como evidencia autosuficiente de veracidad- que actúa como instrumento privilegiado en la búsqueda de legitimación.

\section{Metodología}

Como hemos apuntado, esta investigación se inscribe en una etnografía multisituada más amplia iniciada en 2014, que aplica el enfoque de "seguimiento del conflicto" (follow the conflict) (Marcus, 1995, pp. 105-110). Siguiendo la propuesta de Bruno Latour basada en las teorías del actor-red, se analiza una controversia mediatizada (Marres y Moats, 2015; Venturini, 2010, 2012), utilizando un enfoque prioritariamente discursivo (Marres, 2015), de orientación socio-semiótica.

Dado que toda controversia es un espacio abierto, en proceso y marcado por la incertidumbre, el estudio de caso reclama creatividad y apertura en la combinación de metodologías y en construcción del corpus (Marres y Moats, 2015), porque difícilmente se podrán establecer a priori unos plazos temporales significativos o una muestra prediseñada en relación a medios y plataformas. Para este estudio de caso se ha aplicado en la construcción del corpus el seguimiento de un grupo de personas y organizaciones (follow the people) y del relato específico que comparten (follow the story), que se analiza a través del rastreo de lógica transmedia desde la obra núcleo del corpus: el documental CHN24H.

El corpus se construye por tanto tomando como obra central el documental Chicas nuevas 24 horas, producido en 2015 y Premio Goya a la mejor película documental $2016^{5}$. El hilo conductor que va a ir vinculando los diversos productos que configuran esta estrategia transmedia, como apuntamos, es la labor y la red de contactos profesionales y personales de su directora Mabel Lozano, quien se ha convertido en una de las voces expertas con mayor peso en el discurso público sobre la trata, desde una posición abolicionista. De esta manera, el recorrido analítico nos conduce, durante una década, desde el corto documental de Lozano en 2007 Voces contra la trata, en colaboración con las organizaciones especializadas como la Asociación para la Prevención, Reinserción y Atención a la Mujer Prostituida (APRAMP) y Proyecto Esperanza, hasta la celebración del Día Internacional contra la trata con fines de explotación sexual en 2017, en el que el Ministerio de Sanidad, Servicios Sociales e Igualdad asume el proyecto CHN24H como propio, con la presentación de la aplicación Happy.

Una vez identificado el corpus y los actores clave, se ha llevado a cabo un análisis semiótico de una muestra de textos compuesta por el documental CHN24H, los sitios web del proyecto CHN24H, las campañas publicitarias de sensibilización, el primer episodio del programa Esclavas y el material de promoción, la guía para periodistas sobre trata de la Comunidad de Madrid y la aplicación Happy. Nos interesan especialmente los elementos paratextuales (trailers, anuncios, portadas, reseñas, etc.)

Producido por Puatarara Films, Hangar Films, 7ArteVital, Aleph Media, Mafalda Entertainment. 
puesto que suelen alcanzar a una audiencia mayor, facilitan los vínculos entre los elementos del proyecto transmedia y por su condensación simbólica permiten identificar la propuesta narrativa e ideológica.

\section{Resultados}

\subsection{Mapeo de productos y actores}

En la Figura 1 se esquematiza el grafo de los componentes ${ }^{6}$ del mundo transmediático, articulado a raíz de CHN24H y que podría funcionar como un mapa de parte de la perspectiva institucional-mediática hegemónica en relación con la controversia sobre prostitución. Aquella que se focaliza en la lucha contra la trata en España y que, directa (o indirectamente), reclama políticas a favor del abolicionismo.

Figura 1. Esquema de relaciones transmedia del proyecto de lucha contra la trata con fines de explotación sexual en España. Fuente: elaboración propia.

\section{0:0 Relaciones transmedia \\ Del proyecto de lucha de la trata con fines de explotación sexual}

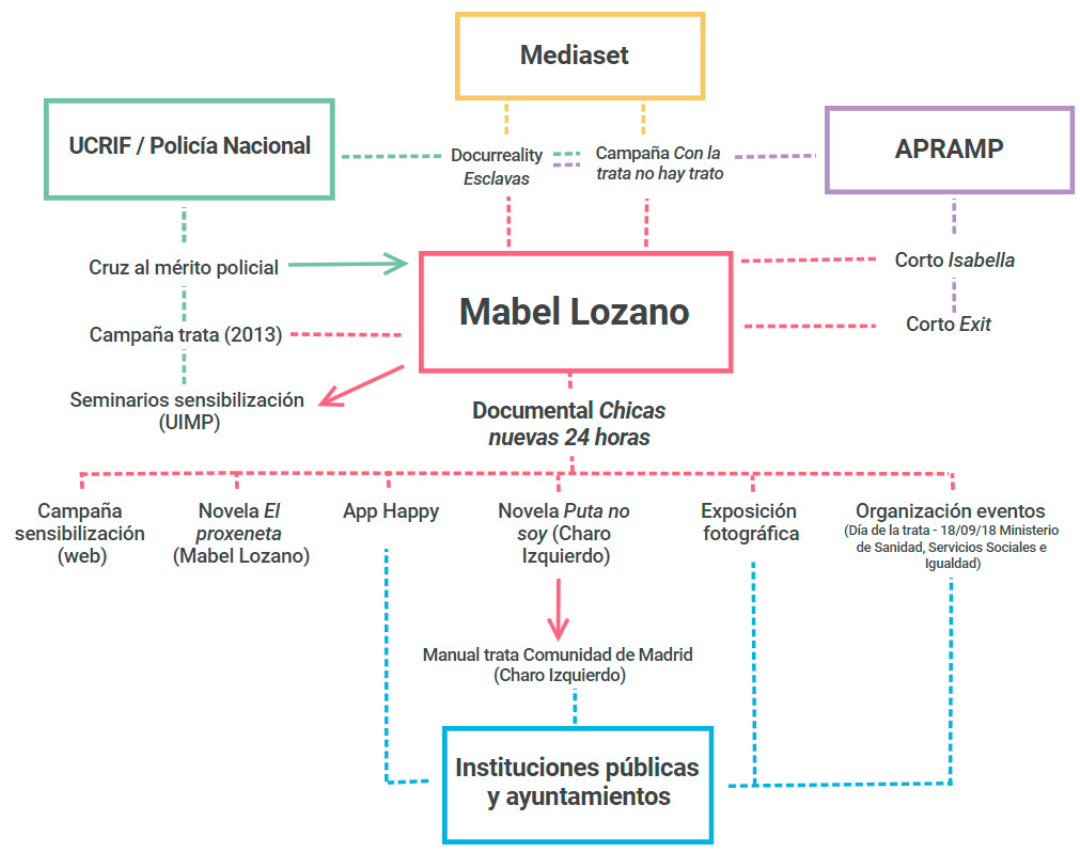

6 Se pueden consultar más datos en: https://figshare.com/s/eeb007e16108cb5a8e49 
Los primeros trabajos de Mabel Lozano marcan una línea de colaboración estable con organizaciones especializadas en la atención de víctimas de trata, especialmente con APRAMP. Comienza en 2007 con el documental Voces contra la trata de mujeres, en colaboración con APRAMP y Proyecto Esperanza; en 2008 realiza el spot promocional de la Red Española contra la trata; en 2010, lanza el corto Escúchame para Médicos del Mundo, que después incluirá en la web de sensibilización del proyecto CHN24H. En 2014, realiza el corto Entre colegas para la Fundación Lydia Cacho, uno de los máximos exponentes de una parte del movimiento abolicionista. En 2014, graba para APRAMP el corto de sensibilización Isabella. Volverá a colaborar con esta entidad en 2016, con el cortometraje Loveth y en 2017, con la campaña Exit.

Tras varios trabajos en colaboración con ONG, en 2013 realiza su primera colaboración con la Policía Nacional grabando el anuncio de la campaña No seas cómplice, el mismo año que recibe la Cruz con distintivo blanco al Mérito Policial. La alianza con esta institución se fragua a través de la relación con José Nieto, responsable de la Unidad Central de Redes de Inmigración Ilegal y Falsedades Documentales (UCRIF). Después, en agosto de 2014, Mabel Lozano participa en el curso celebrado en la Universidad Internacional Menéndez Pelayo (UIMP) "Retos del fenómeno migratorio en el siglo XXI. La trata de seres humanos para la explotación sexual", orientado a miembros de la Policía Nacional y en el que, como representante de organizaciones especializadas, sólo participa APRAMP.

En 2015 se fragua el proyecto $\mathrm{CHN} 24 \mathrm{H}$, que se define como "una acción multidisciplinar" compuesta por un documental, una página web, una exposición fotográfica, un libro, un juego para Smartphone y distintas cuentas en redes sociales.

A raíz de este proyecto, la red de alianzas con otros actores se amplía, así como la producción de nuevos contenidos. La relación entre el proyecto CHN24H y los actores institucionales se forja, en primer lugar, a través de la financiación pública. Entre los principales financiadores del proyecto destacan Acción Cultural Española, la Agencia Española de Cooperación, el Instituto de la Mujer (Gobierno de España) y el Ministerio de Sanidad y Asuntos Sociales. En segundo lugar, la relación se afianza por la estrategia de difusión del documental, dado que no es la usuaria/o final la que está llamada a participar en primera instancia, sino el entramado de instituciones estatales, autonómicas y locales. Resulta significativo, en este sentido, que el documental no se abra a un circuito clásico de comercialización, no esté disponible para su venta, ni se pueda visionar en ninguna plataforma de contenidos, sino que el acceso a público general se realice en su proyección en festivales, pero, sobre todo, a través de actos institucionales, organizados, principalmente, por comunidades autónomas y municipios, de los que se ha dado cuenta en los medios.

Los lazos institucionales son variados: por ejemplo, del documental se deriva la novela Puta no soy, basada en una de las historias de vida, escrita por la periodista Charo Izquierdo. En la presentación de la novela participaron, entre otros, la directora de APRAMP, Mabel Lozano y Cristina Cifuentes, en aquel momento presidenta de la Comunidad de Madrid. Izquierdo redactará posteriormente el manual Cómo informar sobre la trata (2015), financiado por la Comunidad de Madrid y editado por la LID editorial, que publicó la novela de la periodista (Izquierdo, 2015). Además, 
este manual está basado en las recomendaciones realizadas por APRAMP (2015) en su Guía para profesionales de los medios de comunicación.

La posición en la lucha contra la trata de Mabel Lozano como voz hegemónica se consolida apoyada por la relación de su proyecto con los actores institucionales y con los actores mediáticos. Su condición previa como actriz y sus vínculos con la industria mediática le facilitan sus apariciones en los medios y el apoyo institucional le brinda legitimidad. Estos vínculos se consolidan a través de la estrategia de sensibilización compartida entre Mediaset, la Policía Nacional, APRAMP y el equipo de Lozano. La campaña se desarrolló en varias fases: la primera de ellas Contra la trata no hay trato se lanza en 2015, unos años antes que CHN24H, como parte del programa de Responsabilidad Social Corporativa (RSC) de Mediaset 12 meses, 12 causas.

Entre las acciones se incluye, en 2016, el apoyo a la campaña Loveth de APRAMP y la producción del docu-reality Esclavas, emitido en la cadena Cuatro. Ese programa recoge la filosofía de este mundo transmediático, acudiendo como voces expertas a la propia Mabel Lozano, al jefe de la UCRIF, José Nieto, y a la responsable de APRAMP, Rocío Mora, entre otros. Los dos últimos aparecen en el documental CHN24H y son figuras visibles, tanto en la presentación de las campañas de Mediaset como en todos los actos del proyecto. Mediaset también participa en la producción del DVD del documental CHN24H.

La segunda fase del programa de RSC se realiza en septiembre de 2017, La trata es un asunto de todos, firmada también por la Policía Nacional. En la web corporativa de Mediaset se valora la campaña no sólo en términos de impacto en los públicos, sino también en el éxito de la acción derivada ${ }^{7}$, lo que nos sirve como indicio de las interconexiones. La extensión del proyecto $\mathrm{CHN} 24 \mathrm{H}$ ha continuado sobre todo orientado al público más joven, puesto que, en 2016, se crea un rap, se pone a la venta la banda sonora del documental y, por último, en septiembre de 2017 se lanza el juego de móvil Happy.

Actualmente, el proyecto ha mudado su nombre y objetivo y parece que se elaborará una nueva línea transmedia, centrada en la novela que Mabel Lozano acaba de publicar, El Proxeneta, que está basada en la historia de un condenado por trata. José Nieto, responsable de la UCRIF, fue quien puso en contacto a ambos y actualmente está en marcha su adaptación televisiva como programa documental en el canal DKiss: El proxeneta. Paso corto, mala leche. Al igual que sucedió con el documental, el proyecto cuenta con el apoyo y la valoración de las instituciones públicas.

Una década después del primer documental de Lozano, el 23 de septiembre de 2017, el Ministerio de Sanidad, Servicios Sociales e Igualdad organizó, junto con el proyecto CHN24H, los actos de celebración del Día Internacional contra la explotación sexual y el tráfico de mujeres y niños haciendo suya la propuesta discursiva, según se constata en el cartel y en la nota de prensa publicada en la web institucional. Los actores políticos obtienen de esta forma rentabilidad política, al posicionarse en la lucha contra un delito consensuado socialmente y los actores mediáticos, por su parte, consiguen aumentar su reputación en tanto que mediadores sociales, como demuestran los múltiples premios recibidos por Lozano, su equipo y Mediaset.

'Con la trata no hay trato' tuvo un destacado impacto entre los espectadores: durante su primera fase $800 \mathrm{mu}-$ jeres y niñas fueron liberadas de las mafias que las explotaban y la Policía multiplicó por diez las llamadas de denuncia recibidas al 9001050 90. Un teléfono gratuito que no deja rastro en la factura y que ha aparecido constantemente durante toda la campaña. 


\subsection{Análisis de los elementos paratextuales de la estrategia transmedia}

La dimensión paratextual resulta imprescindible en la construcción de la lógica transmedia, puesto que incide en los espacios de recepción y en el consumo cultural. Además, genera expectativas, sintetiza argumentos y tramas y puede favorecer interpretaciones. De acuerdo con Ferrandiz (2015, p. 27), el paratexto funciona como una frontera, "un espacio indeciso entre el interior y el exterior del texto, entre regulaciones de orden propiamente literario, retórico, o estilístico con regulaciones de orden social, económico, industrial, publicitario, una zona transaccional" (Ferrandiz, 2015, p. 27).

En el caso de CHN24H, el mundo transmediático recurre a los elementos paratextuales, en primera instancia, para identificar y categorizar el proyecto como necesario e imprescindible, además de para establecer su autenticidad. Y lo hace no sólo a través de las carátulas, títulos, abstracts o trailers (formas clásicas), sino que hoy se incluyen muchos otros elementos, como los comentarios, las reseñas o las valoraciones, que acompañan a los productos de la red transmedia y favorecen su extensión y consolidación.

Esta apelación a la "necesidad" de los productos que forman parte del entramado transmedia no es garantía, sin embargo, de la calidad de algunos de ellos. Un ejemplo es la aplicación Happy, que, en forma de juego interactivo, está destinado en principio a un público adolescente. La app reproduce los "ganchos" narrativos que aparecen en todo el entramado transmedia. En el juego, Happy es una chica nigeriana víctima de una red de trata. El usuario/a, entre otras acciones, ha de "salvarla" evitando que caiga al mar, mientras lo atraviesa en una balsa de plástico. Algo que se consigue accionando unas flechas que aparecen en pantalla. Gran parte de los comentarios de valoración de usuarias/os de esta aplicación en el Play Store son negativos, sobre todo los que tienen que ver con el planteamiento narrativo y la jugabilidad. Sin embargo, se justifica y apoya la existencia de la app en función de su utilidad social, como si cualquier producto que se desarrolle en esta línea temática pudiera tener capacidad sensibilizadora en sí mismo, por el hecho de tratar un tema de controversia social. Creemos que es necesario un análisis crítico de las estrategias retóricas que en ocasiones envuelven a proyectos, que se configuran desde lo tecnológico tan sólo a partir de una idea-núcleo de éxito, pero que no contribuyen significativamente a la innovación social sobre un problema, puesto que la mera participación "interactiva" no es autosuficiente.

También a través de los elementos paratextuales, el mundo transmediático argumenta y refuerza su legitimidad recurriendo al realismo y a la retórica de la autenticidad, a veces para salvar las limitaciones que puede imponer el uso de la ficcionalización del testimonio. La app Happy, por ejemplo, es presentada en la web del proyecto como "una aventura basada en una historia real de una víctima de trata nigeriana que llegó a España engañada para ser explotada sexualmente". En la Play Store se reitera la idea: “¡Nunca has participado en un juego así! Es la primera vez que tienes en tu mano una historia real contada en formato videojuego para móviles, que te llevará a las raíces de un delito internacional, que no es otra cosa que una violación de los derechos humanos: la trata de personas".

La apelación al realismo como garantía de autenticidad acaece incluso en eventos institucionales, como en el curso "Retos del fenómeno migratorio en el siglo XXI. La trata de seres humanos para explotación sexual", organizado por la Universidad Internacional Menéndez Pelayo en el año 2014. En el programa de este curso se lee: 
"Mesa redonda. Testimonio real de una víctima". Mientras el resto de ponentes de la mesa están identificados con sus nombres y cargos institucionales, en el caso de la participante afectada se la describe como "víctima", destacando que lo que ofrece es un "testimonio real", algo que resulta, por ende, redundante.

En la contraportada de la carátula del DVD CHN24H se hace, de nuevo, alusión al realismo a través del siguiente testimonio, que acompaña a la fotografía de una chica joven:

Intenté escapar de un horror que ningún ser humano puede imaginar. Ellos me cogieron, y a modo de escarmiento para mí y el resto de las mujeres, me pegaron muy duro, me rasuraron las cejas y el cabello, y me ataron a un radiador como se ata a los perros. Después, con un cuchillo me tatuaron mi deuda en la muñeca." (Fotografía real de Madilla, 20 años, víctima de trata, rescatada en Madrid por la Policía Nacional).

El docu-reality Esclavas, por su parte, buscaba "recrear casos reales" de la "lacra" de la trata, y "narrar la realidad de la prostitución en nuestro país, un negocio que ejercen 45.000 mujeres y que mueve 5 millones de euros al día, y centrar nuevamente el foco de la concienciación social sobre la trata de mujeres"; lo que nos permite comprobar cómo se va reforzando y construyendo la legitimidad del entramado transmedia mediante esa apelación a lo real, a lo auténtico.

\section{Discusión: mediatización de la controversia y lógica transmedia}

La mediatización se define como el conjunto de las transformaciones estructurales producidas en la intersección entre los medios, la cultura y la sociedad, que suceden predominantemente dentro de instituciones, dominios o campos sociales particulares (Hjarvard, 2016). Esto presupone que la mediatización "no sólo conduce a una mayor presencia de los medios de comunicación en la cultura y en la sociedad, sino que también motiva que otras instituciones y ámbitos dependan de ellos y de sus lógicas" (Hjarvard, 2016, p. 240). Hjarvard defiende que hay una conexión estructural entre las dinámicas institucionales y mediáticas, así como con las formas de acción e intervención pública. Los medios se interpretan "como herramientas sociales" y en este sentido "pueden permitir, limitar y estructurar las maneras en las que la acción y la interacción se desarrollan" (Hjarvard, 2016, p. 41).

La mediatización debe adaptarse a contextos particulares (ibid., 46) y en un contexto de controversia significa que se usarán lógicas mediáticas particulares, definidas estas como un conjunto de procedimientos convencionalizados, caracterizados por dimensiones técnicas, estéticas e institucionales, es decir, compuestas por "reglas (formales e informales) y recursos (materiales y autoritativos) institucionales" (ibid., 241-242). Una de ellas es la lógica transmedia, de la que hemos expuesto un ejemplo a través del estudio de caso CHN24H. El modelo clásico de lógica mediática para la incidencia social y política basado en la atención y publicación en los medios mainstream de una perspectiva -por ejemplo, a través de comunicados y notas de prensa- resulta hoy insuficiente. Los actores en una controversia combinan 
estrategias más complejas tanto de producción propia, como de búsqueda de apoyo mediático para generar sinergias entre sí y aprovechar el contexto de convergencia.

La EPM está fragmentada, traspasada por múltiples fronteras y compuesta por diversidad de arenas públicas en las que participar, conectarse, hacer circular propuestas y construir posiciones de poder (o contrapoder) en relación a los problemas públicos (Cefaï, 2011; Peñamarín Beristain, 2017). En este contexto, la lógica transmedia permite estabilizar arenas públicas, al promover y catalizar las alianzas de actores, dotar de coherencia y continuidad a todos los productos de diferentes géneros discursivos que componen una perspectiva y permitir que esta adquiera una posición identificable, consolidada y legitimada por su carácter común y compartido. Gracias a esta escenificación múltiple les resulta más sencillo evitar que su propuesta se identifique con un punto de vista particular y estarán más cerca de componer un mundo transmediático (Klastrup y Tosca, 2004) que se acabe admitiendo como una versión de sentido común sobre el asunto que se aborda.

En el caso que hemos analizado, este mundo con una perspectiva particular (la abolicionista) se proyecta y conecta con valores, disposiciones afectivas y lugares comunes compartidos, fuertemente asidos en los imaginarios y enciclopedias colectivas. Por ejemplo, en estos discursos se identifica la prostitución como el oficio más antiguo y, por tanto, como una institución patriarcal atemporal que no ha sufrido modificaciones. Se presupone una degradación moral a los intercambios de sexo por dinero (Zelizer, 2011) o se conjetura la existencia de una masculinidad violenta implícita en todos los intercambios sexuales de este tipo.

Así mismo, se utilizan argumentos ideológicos sobre el asunto controvertido que sirven para sostener posiciones políticas que, al mismo tiempo, refuerzan la credibilidad y legitimidad de la posición de partida. Por ejemplo, el proyecto $\mathrm{CNH} 24 \mathrm{H}$, al recurrir profusamente a fuentes institucionales y promover un relato de la trata centrado en la intervención de los expertos como vía de solución -casi única- frente a las condiciones de vulnerabilidad, colabora en la legitimación de dispositivos de control propios de la biopolítica. Estos, sin embargo, han sido fuertemente contestados desde otras perspectivas por sus efectos negativos, como se puede ver en el cuestionamiento sobre la industria del rescate (Agustin, 2007, pp. 191-193) o las críticas a los modelos de securitización sobre la trata (Grundell, 2017).

A través de un relato expandido, reiterado y cohesionado, el proyecto $\mathrm{CHN} 24 \mathrm{H}$ configura un horizonte de expectativas (axiológico, ideológico y afectivo), que modela las normas de interpretación aplicables al fenómeno de la trata -y por extensión a la prostitución. Gracias a la lógica transmedia descrita podría considerarse que el proyecto $\mathrm{CHN} 24 \mathrm{H}$ coloniza los espacios ciudadanos y se erige en buque insignia de una perspectiva, adoptada como discurso propio por muchas voces institucionales. Si el Estado a través de sus instituciones asume una cierta alianza con algunos discursos activistas, se transforma en aliado, en este caso, de una parte del movimiento abolicionista. En este proceso, los agentes institucionales acreditan y autorizan a otros actores, aumentando su representatividad socio-política, mientras se excluye o desacredita a otros.

Gracias a esta red de colaboración, los actores sociales que defienden la perspectiva hegemónica se arrogan el estatuto privilegiado de la mediación, al menos en los espacios de la esfera pública central, y además será hacia ellos a donde se canalicen los recursos. El Estado "remodela entonces el espacio de la protestación colectiva, calmando a unos y agitando a otros, dándose interlocutores oficiales, al mismo tiempo 
que priva de voz a sus competidores, asegurándose gracias a redistribuciones diversas del consentimiento de la mayoría y gestionando el espacio público mientras mantienen el control tanto como pueden" (Cefaï, 2011, p. 146).

No estamos argumentado que las conexiones señaladas respondan a motivaciones exclusivamente economicistas, empresariales o de rédito electoral, pero sí de la existencia de un handicap: en la controversia los actores de una perspectiva han obtenido el favor del entramado de actores institucionales, dificultando, cuando no bloqueando, la discusión, al ubicarla en el terreno incontrovertible de la lucha contra la trata. En este contexto, hay otros actores, por ejemplo el movimiento en defensa del trabajo sexual que, al mismo tiempo, procuran hacer valer su punto de vista sobre la prostitución y los conflictos asociados. Estos comparten también la necesidad de luchar contra el delito, respetar los derechos de mujeres y niñas y ofrecerles protección, pero su perspectiva sobre el asunto es diversa, al igual que sus propuestas de solución. En la deliberación que se produce en la EPM se ponen a consideración pública los sentidos que articulan la moral del tiempo y espacio en que dichas representaciones son posibles. Nuestros análisis indican que los relatos e imaginarios hegemónicos en los medios vinculados al abolicionismo, ilustrados por productos como $\mathrm{CHN} 24 \mathrm{H}$, están tornándose homogéneos y articulándose desde posiciones incontrovertibles que dificultan la discusión particular sobre medidas y obstaculizan la consecución de acuerdos.

Desde un punto de vista narrativo, la cuestión de la trata está planteada desde un punto de vista centrado en el delito y, hasta el momento, con un enfoque victimocéntrico. Esta focalización opera hipervisibilizando este aspecto, mientras se ensombrecen otros. Se abriga en la retórica de las grandes cifras y en el estado de alarma. Remitir al delito funciona como estrategia para negar la posibilidad del trabajo sexual, o la de los fenómenos sociales que se encuentran en zonas grises y no pueden subsumirse como trata con fines de explotación sexual. Ubicarse en el consenso facilita concebir la perspectiva de lucha contra la trata desde la restricción de la demanda (y, por tanto, la necesidad de abolición de la prostitución) como única solución legítima, acudiendo, entre otros argumentos, a la equiparación entre trata y prostitución.

Se expande un relato reconocible, articulado sobre un testimonio que reconstruye la narración estereotípica válida para todas y en todos los contextos geográficos, económicos y culturales: mujer desempoderada, engañada, víctima de su entorno cultural, sociopolítico y familiar, propios de países empobrecidos, explotada por redes de malvados - tratantes inespecíficos- y por la actuación de violentos demandantes de prostitución, que, finalmente, es rescatada por la policía y atendida por las instituciones del Estado.

A falta de estudios más pormenorizados, creemos que el testimonio, tal y como se usa en CHN24H, no busca destacar la especificidad de cada caso, sino la base de todo aquello que los testimonios tienen de común entre sí y que capacita para invisibilizar las diferencias culturales, geográficas, políticas y/o de clase, así como las diferencias de las trayectorias migratorias, entre otros aspectos.

Contrasta en este sentido que las mujeres protagonistas sean identificadas solamente con el nombre propio, sin filiación individual y tan solo con un origen genérico (nacional), mientras que las voces expertas están formalmente establecidas. Este uso del testimonio podría estar impidiendo poner de manifiesto una visión política del fenómeno, una densificación del discurso capaz de señalar los conflictos y para- 
dojas que afectan al fenómeno de la trata y a las políticas públicas para su erradicación, así como la visibilización de críticas a las autoridades.

Al promover una representación homogénea y fetichizada de la prostitución y de las víctimas de trata se dificulta el testimonio ético, porque no se apunta a la responsabilidad colectiva, ni a los aspectos estructurales, ni se trabaja en la reparación del daño (Gámez Fuentes y Gómez Nicolau, 2017). Frente a ello, las mujeres que forman parte del movimiento de trabajo sexual reivindican que sus testimonios no se ficcionalicen, ni se subsuman sus experiencias en un relato homogéneo adecuado a una perspectiva colonial del mundo. Reclaman no ser una oportunidad para el heroísmo por parte de las instituciones y alegan su derecho a tener trayectorias múltiples, híbridas e inasibles.

No hemos detectado en el mundo transmediático que representa el proyecto CHN24H la puesta en marcha de una práctica comprometida en un sentido fuerte con la propuesta del testimonio ético. En este ejercicio de escucha habría que resolver mejor la cuestión de la responsabilidad sobre la trata, vinculándola a las estructuras económicas que generan desigualdad y a las políticas públicas que pueden estar agudizando el problema, ya sean las normas locales, nacionales o europeas, sobre convivencia ciudadana, seguridad, migraciones, etc. Cabría desplazar el lugar de enunciación desde la superioridad moral, retóricamente construida en defensa de las víctimas, hacia una posición de incertidumbre y de cuestionamiento que abra espacio a la reflexión y a la empatía desde la deuda con las otras reconocidas como inapropiables. Y concebir los productos mediáticos como herramientas de reparación, no orientándolos únicamente a públicos generales, sino tomando a estas otras, no solo como fuentes sino como públicos afectados directamente por los relatos y las representaciones que circulan en la EPM.

\section{Conclusiones}

Mediante el análisis etnográfico del problema público de la trata con fines de explotación sexual y del asunto sobre la prostitución hemos detectado la importancia de los diferentes géneros de discurso en la conformación y en el desarrollo de las controversias. La estabilización de un asunto y la institucionalización de arenas y actores va a depender en cierta medida de las estrategias de mediatización. Cada controversia moviliza dinámicas diversas: si en los asuntos científicos será central la producción de información especializada y la divulgación, en las cuestiones sociales que forman parte de las enciclopedias comunes -como la prostitución-, el objetivo es atraer la atención de los públicos desde el territorio de los lugares comunes y las disposiciones afectivas compartidas.

La lógica transmedia se adapta bien a este supuesto ya que favorece la construcción de un mundo a partir de actores, cronotopos y valores cohesionados por un relato mítico con múltiples puntos de acceso, ubicados en diferentes arenas públicas. Este mundo transmediático ofrece la posibilidad a diferentes actores -institucionales, mediáticos, activistas- tanto de vincularse como de colaborar en su articulación en los términos que mejor se adapten a sus condiciones, intereses y expectativas. Por otra parte, dado que se trata de una lógica de mediatización que requiere una alta inversión de recursos (simbólicos, sociotécnicos y económicos) será una estrategia especialmente útil para los actores ya institucionalizados o en alianza con estos que podrán reforzar su posición hegemónica, como sucede en el caso de estudio. 
El proyecto $\mathrm{CHN} 24 \mathrm{H}$ ha logrado un elevado impacto en la EPM adaptándose a esta lógica transmedia, captando recursos a través de un relato que permite a las instituciones desplegar su condición privilegiada de expertos, en consonancia con la figura estereotipada de la víctima (vulnerable y sin agencia), todo ello reforzado por una portavocía personalista que se adapta bien al modelo de celebrity mediática. La promoción de una postura argumentativa consensual, defendida desde una premisa incontrovertible (la trata es un delito y una vulneración de los derechos humanos que debe combatirse), unida a los vínculos profesionales y personales derivados del proyecto, han facilitado el cruce de su propuesta discursiva desde el activismo, con los intereses, objetivos y discursos político-institucionales y de los medios mainstream. No obstante, el análisis preliminar de la propuesta narrativa y retórica muestra un bajo nivel de innovación social y ciertas limitaciones para considerarlo como una propuesta de testimonio ético sobre la trata con fines de explotación sexual, puesto que esto implicaría poner en primer lugar, entre otros aspectos, el ejercicio de representación como acto de reparación del daño y la injusticia sufrida por las víctimas, apostando por la profundización y apertura de los sentidos frente a la simplificación y reducción. Específicamente, en el contexto de una controversia pública supondría además dar herramientas para afrontar la incertidumbre y reconocer el disenso como herramienta en la búsqueda de soluciones.

\section{Referencias}

Agustin, L. (2007). Sex at the margins. London: Zed Books.

Andrijasevic, R., y Mai, N. (2016). Editorial: Trafficking (in) Representations: Understanding the recurring appeal of victimhood and slavery in neoliberal times. Anti-Trafficking Review, 7, 1-10. https://doi.org/https://doi.org/10.14197/atr.20121771

APRAMP (2015). Guía de Intervención con Victimas de Trata para Profesionales de los Medios de Comunicación. Madrid: APRAMP. Disponible en https://goo.gl/mG7xnb

Cefaï, D. (2011). Diez propuestas para el estudio de las movilizaciones colectivas. De la experiencia al compromiso. Revista de Sociología, 26, 137-166. Disponible en https://goo.gl/f4zxTq

Cefaï, D. (2016). Publics, problèmes publics, arènes publiques: que nous apprend le pragmatisme? Questions de Communication, 30, 25-64.

Comunidad de Madrid (2015). Cómo informar sobre la trata. Manual de estilo para medios de comunicación. Madrid: LID Editorial. Disponible en http://www.madrid.org/bvirtual/ BVCM013952.pdf

Devillard, V., y Le Saulnier, G. (2015). Le problème public de la prostitution aux marges des arènes publiques numériques. Luttes interprétatives et mobilisations pro-droits contre l'offensive abolitionniste sur le web. Journal des anthropologues, 142-143(3-4), 203226. Disponible en https://www.cairn.info/load_pdf.php?ID_ARTICLE=JDA_142_0203

Ferrandiz, R. R. (2015). El cuento de nunca acabar: precuelas y secuelas de la narración transmedia. En R. Rodríguez y V. Tur (Eds.), Narraciones sin fronteras Transmedia storytelling en la ficción, la información, el documental y el activismo social y político (Cuadernos, pp. 21-40). La Laguna (Tenerife): Sociedad Latina de Comunicación Social. Disponible en http://www.cuadernosartesanos.org/2015/cac81.pdf

Gámez Fuentes, M. J., y Gómez Nicolau, E. (2017). El 'testimonio ético' como modelo analítico en la comunicación para el cambio social. Atas CIAIQ 2017 Investigaçao Qualitativa Em Ciencias Sociais, 3, 170-179. Disponible en https://goo.gl/Bescvt 
Gimeno, B. (2012). La prostitución. Aportes para un debate abierto. Barcelona: Ediciones Bellaterra. Gobierno de España (2018). Pacto de Estado contra la Violencia de Género (Texto refundido). Disponible en https://goo.gl/LwS8sH

Grundell, L. (2017). Repensar la relación entre seguridad y ciudadanía: La regulación europea de la trata de mujeres con fines de explotación sexual como caso de estudio. Relaciones Internacionales, 35, 53-78. https://doi.org/10.15366/relacionesinternacionales2017.35.003

Gusfield, J. (1991). The culture of public problems. Communities of Fate, Readings in the Social Organization of Risk.

Heim, D. (2012). Más allá del disenso: los derechos humanos de las mujeres en los contextos de prostitución. Derechos y Libertades: Revista de Filosofía Del Derecho y Derechos Humanos, 26, 297-327. http://dx.doi.org/10.1400/216303

Hjarvard, S. (2016a). Mediatización: La lógica mediática de las dinámicas cambiantes de la interacción social. La Trama de La Comunicación, 20(1), 235-252. Disponible en https:// goo.gl/8Q9uua

Hjarvard, S. (2016b). Mediatización: reencuadrando el análisis de los efectos de los medios. InMediaciones de La Comunicación, 11, 33-56. Disponible en https://goo.gl/xpEMtR

Izquierdo, C. (2015). Puta no soy. Madrid: LID Editorial.

Jenkins, H. (2011). Transmedia 202: Further Reflections. Confessions of an Aca-Fan. Henry Jenkins Blog. Disponible en http://henryjenkins.org/blog/2011/08/defining_transmedia further_re.html

Juliano, D. (2005). El trabajo sexual en la mira: polémicas y estereotipos. Cadernos PaguMercado Do Sexo. https://doi.org/10.1590/S0104-83332005000200004

Justo von Lurzer, C. (2014). Sexualidades en foco. Representaciones televisivas de la prostitución en la Argentina. Sexualidades. Center for Lesbian and Gay Studies Graduate Center, 11, 1-57. Disponible en http://hdl.handle.net/11336/35849

Kapur, R. (2002). The Tragedy of Victimization Rhetoric: Resurrecting the "Native" Subject in International/Post-Colonial Feminist Legal Poltiics. Harvard Human Rights Journal.

Klastrup, L., y Tosca, S. (2004). Transmedial worlds - Rethinking cyberworld design. In Proceedings - 2004 International Conference on Cyberworlds, CW 2004. https://doi. org/10.1109/CW.2004.67

Lozano, M. (2017). El proxeneta. Barcelona: Alreves.

Marcus, G. E. (1995). Ethnography in / of the world system. The Emergence of Multi-Sited Ethnography. https://doi.org/10.1177/1463499605059232

Marres, N. (2015). Why Map Issues? On Controversy Analysis as a Digital Method. Science Technology and Human Values. https://doi.org/10.1177/0162243915574602

Marres, N., y Moats, D. (2015). Mapping Controversies with Social Media: The Case for Symmetry. Social Media + Society, 1(2). https://doi.org/10.1177/2056305115604176

Núñez Puente, S., y Fernández Romero, D. (2015). Identity construction of the subject victim of domestic violence: fetishism, aesthetization and public identity. Teknokultura, 12(2), 267-284. http://dx.doi.org/10.5209/rev_TK.2015.v12.n2.49506

Patte, Y. (2006). On the Notion of "Field" [of Investigation]: The "Geometric Manner" Approach to a Public Debate — Prostitution in Belgium. Sociologie et Societes, 38(1), 235-261. http://dx.doi.org/10.7202/013716ar

Peñamarín Beristain, C. (2017). Mediación y mediatización de la comunicación pública. Una perspectiva pragmática. In M. Alvarez-Peralta, G. Fernandez, y L. Mazolli (Eds.), a mediación fragmentaria Mediatización y controversia en la nueva esfera pública (pp. 11-36). Cuadernos Latina. Disponible en https://goo.gl/FxDMZ4 
Puñal Rama, B., y Tamarit, A. (2017). La construcción mediática del estigma de prostituta en España. Ex Aqquo, 35, 101-123. https://doi.org/10.22355/exaequo.2017.35.07

Rodríguez Ferrándiz, R., y Peñamarín, C. (2014). Narraciones transmedia y construcción de los asuntos públicos. Introducción. CIC Cuadernos de Información y Comunicación. https://doi.org/10.5209/CIYC.43900

Sabsay, L. (2016). The political imaginary of sexual freedom. London: Palgrave, Macmillan.

Saiz-Echezarreta, V., Alvarado, M., y Gomez Lorenzini, P. (2018). Incidencia política de las campañas contra la trata: Un relato controvertido. Revista Comunicar, 55. https://doi.org/ https://doi.org/10.3916/C55-2018-03

Sanders, T., O’Neill, M., \& Pitcher, J. (2009). Prostitution. Sex work, policy y politics. Los Angeles: SAGE Publications

Serughetti, G. (2018). Rethinking force and consent, victimisation and agency: a feminist approach to prostitution policy. Femeris, 3(2), 79-107. https://doi.org/https://doi. org/10.20318/femeris.2018.4321

Venturini, T. (2010). Diving in magma: How to explore controversies with actor-network theory. Public Understanding of Science. https://doi.org/10.1177/0963662509102694

Venturini, T. (2012). Building on faults: How to represent controversies with digital methods. Public Understanding of Science. https://doi.org/10.1177/0963662510387558

Wagenaar, H. (2017). Why Prostitution Policy (Usually) Fails and What to Do about It? Social Sciences, 6(2). https://doi.org/10.3390/socsci6020043

Zelizer, V. A. (2011). Economic Lives: How Culture Shapes the Economy. New Jersey: Princeton University Press. 